\title{
6th International Workshop on Human Activity Sensing Corpus and Applications (HASCA)
}

\section{Kazuya Murao}

Ristumeikan University, Japan

murao@cs.ritsumei.ac.jp

\section{Yu Enokibori}

Nagoya University, Japan

enokibori@i.nagoya-u.ac.jp

Hristijan Gjoreski

Ss. Cyril and Methodius

University, Macedonia

hristijang@feit.ukim.edu.mk

\section{Paula Lago}

Kyushu Institute of Technology,

Japan

lago.paula-

andrea346@mail.kyutech.jp

Paste the appropriate copyright statement here. ACM now supports three different copyright statements:

ACM copyright: ACM holds the copyright on the work. This is the historica approach

License: The author(s) retain copyright, but ACM receives an exclusive

publication license.

Open Access: The author(s) wish
additional fee must be paid to $A C M$ single spaced in a sans-serif 7 point font.

Every submission will be assigned their own unique DOI string to be included here.

\begin{abstract}
The recognition of complex and subtle human behaviors from wearable sensors will enable next-generation humanoriented computing in scenarios of high societal value (e.g. dementia care). This will require large-scale human activity corpuses and much improved methods to recognize activities and the context in which they occur. This workshop deals with the challenges of designing reproducible experimental setups, running large-scale dataset collection campaigns, designing activity and context recognition methods that are robust and adaptive, and evaluating systems in the real world. We wish to reflect on future methods, such as lifelong learning approaches that allow open-ended activity recognition.
\end{abstract}

Unique this year, HASCA will welcome papers from participants to the Sussex-Huawei Locomotion and Transportation Recognition Competition in a special session.

\section{Author Keywords}

Large Scale Human Activity Sensing Corpus, Activity Recognition, Wearable Computing, Open-Ended Activity/Context Recognition, Smartphones, SHL Activity Recognition competition, Mobile Sensors, Participatory Sensing. 


\section{Objective of the Workshop}

The objective of the workshop is to bring together researchers and practitioners both from academia and industries with the goal to discuss, identify and share experiences surrounding the construction of human activity sensing corpuses and their applications. Similarly to other humanrelated information processing areas, such as speech recognition and image recognition, real-world activity recognition requires large-scale corpuses of data. Some initiatives set reference datasets for the use by the community, such as OPPORTUNITY [2], HASC [5], or SHL [3] but more work on larger scale and richer datasets are clearly required. Scenarios of high societal value - such as a memory prosthesis for people with dementia - are likely to require a more complete and subtle understanding of the user's activities and the context in which they occur, beyond what is currently available in "off the shelf" datasets. Similarly, activity recognition methods will have to be further improved to tackle real-world challenges.

As in previous years, this year we will have invited talks and a standard call for individual research results related to workshop topics.

This year, the workshop will include a session dedicated to the Sussex-Huawei Locomotion and Transportation Activity Recognition Competition [1, 3]. The SHL Activity Recognition competition is organized by the HASCA organizers using a the University of Sussex-Huawei Locomotion (SHL) dataset [1], which is a large-scale dataset for multimodal locomotion analysis collected during 2017. The competition will run few months before the workshop. The participants will be encouraged to submit a paper for the HASCA workshop to present their results, and we will unveil during the workshop which is the best performing algorithm.
In addition, we are looking to evolve the workshop style towards increasing collaborations within the community on larger scale tasks and challenges. Many domains have successed with such community-wide collaborations, e.g., collecting joint datasets accross researchers all over the world, using the same sensors, targetting the same tasks, and harmonizing these results. One example is the CVPR challenge. At the end of the workshop, the participants will discuss the next year's collaborative work based on the entered projects and aim for signifficant improvements of HASCA for the next year.

\section{Topics of the Workshop}

The objective of this workshop is to share the experiences among current researchers around the challenges of realworld activity recognition, the role of datasets and tools, and breakthrough approaches towards open-ended contextual intelligence. We expect the following domains to be relevant contributions to this workshop (but not limited to):

1. Data collection / Corpus construction: Experiences or reports from data collection and/or corpus construction projects, such as papers describing the formats, styles or methodologies for data collection. Cloudsourcing data collection or participatory sensing also could be included in this topic.

2. Effectiveness of Data / Data Centric Research: There is a field of research based on the collected corpus, which is called "Data Centric Research". Also, we solicit of the experience of using large-scale human activity sensing corpus. Using large-scape corpus with machine learning, there will be a large space for improving the performance of recognition results.

3. Tools and Algorithms for Activity Recognition: If we have appropriate and suitable tools for management 
of sensor data, activity recognition researchers could be more focused on their research theme. However, development of tools or algorithms for sharing among the research community is not much appreciated. In this workshop, we solicit development reports of tools and algorithms for forwarding the community.

4. Real World Application and Experiences: Activity recognition "in the Lab" usually works well. However, it is not true in the real world. In this workshop, we also solicit the experiences from real world applications. There is a huge gap/valley between "Lab Environment" and "Real World Environment". Large scale human activity sensing corpus will help to overcome this gap/valley.

5. Sensing Devices and Systems: Data collection is not only performed by the "off the shelf" sensors. There is a requirement to develop some special devices to obtain some sort of information. There is also a research area about the development or evaluate the system or technologies for data collection.

6. Mobile experience sampling, experience sampling strategies: Advances in experience sampling approaches, for instance intelligently querying the user or using novel devices (e.g. smartwatches) are likely to play an important role to provide user-contributed annotations of their own activities.

7. Unsupervised pattern discovery: Discovering meaningful repeating patterns in sensor data can be fundamental in informing other elements of a system generating an activity corpus, such as inquiring user or triggering annotation crowd sourcing.

8. Dataset acquisition and annotation through crowdsourcing, web-mining: A wide abundance of sensor data is potentially in reach with users instrumented with their mobile phones and other wearables. Capitalizing on crowd-sourcing to create larger datasets in a cost effective manner may be critical to open-ended activity recognition. Online datasets could also be used to bootstrap recognition models.

9. Transfer learning, semi-supervised learning, lifelong learning: The ability to translate recognition models across modalities or to use minimal supervision would allow to reuse datasets across domains and reduce the costs of acquiring annotations.

\section{Workshop Format}

There will be a keynote talk by a practitioner in the field who can provide their opinion of successes and failures in the field of human activity recognition, in order to set the tone for the workshop. Additionally, there will be a special session for the best teams at the SHL Activity Recognition challenge, which will be organized by the HASCA organizers before the workshop, during June-July. There will be a discussion session after all of the presentations. The aim is to avoid being a mini-conference but instead provide a forum within which to explore the issues in human activity sensing corpus, and begin setting a road map for future research and collaboration in data collection and tools development. The intention of this discussion session is to provide early feedback to the presenters on their ideas, and primarily to engender discussion of what approaches in general the participants consider promising. In short, we plan up to three technical paper sessions, keynote talk, and up to one special session for the SHL Activity Recognition challenge in the one-day workshop. 


\section{Estimated number of participant}

We expect to bring together 40-50 participants who are working on or having interest with human activity sensing corpus and its applications (last year's participant count was over 40 [4]). Our workshop is open to anyone and we do not cap the number of participants because it is very important to share the experience and the information of the corpus in this field for the real-world practical applications.

\section{Publication}

All contributions will be included in the ACM Digital Library and supplemental proceedings of the conference. Our website will be http://hasca2018.hasc.jp/

\section{Prospective outcome}

The workshop will provide knowledge about current stateof-the-art studies around human activity recognition with open-ended concept for UbiComp/ISWC community. Expected outcomes are:

1. Survey of the state of the art of "Human Activity Sens ing Corpus". This includes an overview of the data collection methods, tools and algorithms.

2. Practical knowledge of the data collection methodologies for human activity sensing.

3. Recognition of the potential and the importance of the large-scale corpus for human activity recognition.

\section{Pre-workshop activities}

We have already held the past five HASCA workshops from 2013 through 2017. Every time, there are new attendees and the community has been growing up continuously.

\section{Post-workshop activities}

On the workshop website, the list of the current data collection activities and the information of the public corpora will be presented. We will provide a mailing list for the community.

\section{Organizers}

The most of the organizers are from OPPORTUNITY [2] and HASC [5] group.

\section{REFERENCES}

1. 2017. The University of Sussex-Huawei Locomotion (SHL) dataset and competition.

http://www.shl-dataset .org/activity-recognition-challenge/

2. M. Rossi T. Holleczek G. Tröster P. Lukowicz G. Pirkl D. Bannach A. Ferscha J. Doppler C. Holzmann M. Kurz G. Holl R. Chavarriaga H. Sagha H. Bayati D. Roggen, A. Calatroni and J. del R. Millán. 2010. Collecting complex activity data sets in highly rich networked sensor environments. In INSS '10.

3. L. Wang F. J. O. Morales S. Mekki S. Valentin D. Roggen H. Gjoreski, M. Ciliberto. 2018 [In Print]. The University of Sussex-Huawei Locomotion and Transportation Dataset for Multimodal Analytics with Mobile Devices. In IEEE Access.

4. D. Roggen S. Inoue S. Pirttikangas K. V. Laerhoven N. Kawaguchi, N. Nishio. 2017. 5th Int. workshop on human activity sensing corpus and applications (HASCA): towards open-ended context awareness. In UbiComp 2017 Adjunct Publication. 530-536.

5. T. Yang N. Ogawa Y. Iwasaki K. Kaji T. Terada K. Murao S. Inoue Y. Kawahara Y. Sumi N. Nishio N. Kawaguchi, Y. Yang. 2011. HASC2011corpus: Towards the Common Ground of Human Activity Recognition. In UbiComp2011. 571-572. 